\title{
Anaplastic carcinoma of the pancreas arising in an intraductal papillary mucinous neoplasm: A case report
}

\author{
KENSUKE FUJII $^{1}$, TOSHIKATSU NITTA ${ }^{1}$, HIROSHI KAWASAKI ${ }^{1}$, JUN KATAOKA ${ }^{1}$, TOMO TOMINAGA ${ }^{1}$, \\ YOSHIHIRO INOUE ${ }^{2}$, EIJI KADOTA ${ }^{3}$, TAKASHI ISHIBASHI ${ }^{1}$ and KAZUHISA UCHIYAMA ${ }^{2}$ \\ ${ }^{1}$ Department of Gastroenterological Surgery, Shiroyama Hospital, Habikino, Osaka 583-0872; \\ ${ }^{2}$ Department of General and Gastroenterological Surgery, Osaka Medical College Hospital, Takatsuki, Osaka 569-0801; \\ ${ }^{3}$ Division of Pathology, Japan Clinical Laboratories, Inc., Moriguchi, Osaka 570-0033, Japan
}

Received June 8, 2015; Accepted October 30, 2015

DOI: $10.3892 / \mathrm{mco} .2015 .671$

\begin{abstract}
We herein report a case of anaplastic carcinoma of the pancreas arising in an intraductal papillary mucinous neoplasm (IPMN). A 68-year-old Japanese woman was admitted to our hospital complaining of fatigue. Computed tomography revealed an irregular mass in the pancreatic head, which displayed high-signal intensity on diffusion-weighted magnetic resonance imaging. Accordingly, the patient was diagnosed with pancreatic cancer and underwent pancreaticoduodenectomy. The histopathological findings revealed intraductal papillary proliferative changes involving the main and branch ducts of the pancreatic head. Based on the immunohistochemistry results, the intraductal lesion was diagnosed as IPMN. The pathological diagnosis for the invasive carcinoma was anaplastic giant-cell carcinoma of the pancreas (ACP), and the focus of IPMN dedifferentiation to ACP was found to be located at the periphery of the IPMN. At 18 months postoperatively, the patient remains disease-free.
\end{abstract}

\section{Introduction}

Anaplastic carcinoma of the pancreas (ACP) is a rare undifferentiated tumor accounting for 2-7\% of all exocrine pancreatic tumors (1). The prognosis of ACP is poorer compared with that of common pancreatic ductal adenocarcinoma (PDAC), with a median overall survival of 5.2 months and a 3 -year survival rate of only $3 \%$ (1). Conversely, intraductal papillary mucinous neoplasm (IPMN), which was recently recognized as an epithelial exocrine neoplasm, is associated with a more favorable prognosis compared with common PDAC $(2,3)$. Shon et al (4) previously reported on IPMNs with associated ACP. However, to the best of our knowledge, no case of

Correspondence to: Dr Kensuke Fujii, Department of Gastroenterological Surgery, Shiroyama Hospital, 2-8-1 Habikino, Habikino, Osaka 583-0872, Japan

E-mail: sur149@poh.osaka-med.ac.jp

Key words: anaplastic carcinoma, intraductal papillary mucinous neoplasm
ACP arising in an IPMN has been reported to date. Herein, we report a unique case of a patient with ACP arising in an IPMN.

\section{Case report}

A 68-year-old Japanese woman was admitted to Shiroyama Hospital complaining of fatigue in November, 2013. The laboratory tests showed impaired liver function (aspartate aminotransferase, $147 \mathrm{IU} / \mathrm{l}$; alanine aminotransferase, $189 \mathrm{IU} / \mathrm{l}$; serum amylase, 166 IU/l). A horizontal section on a computed tomography (CT) scan revealed an irregular mass in the pancreatic head exhibiting lower enhancement relative to non-tumor pancreatic parenchyma in the arterial-dominant phase. A coronal section revealed the presence of cysts in the inferior part of the mass, and main pancreatic duct distension by $\sim 11 \mathrm{~mm}$ (Fig. 1A). Magnetic resonance cholangiopancreatography revealed stenosis of the main pancreatic and common bile ducts, caused by the mass-neighboring cysts (Fig. 1B). The mass at the pancreatic head displayed high-signal intensity on diffusion-weighted magnetic resonance imaging (MRI). The CT and MRI scans revealed no evidence of local or distant metastases, and the superior mesenteric vessels were not infiltrated by the tumor. The patient was diagnosed with pancreatic cancer and underwent pancreaticoduodenectomy. Macroscopically, the cut surface of the invasive tumor was solid and whitish yellow, measuring $19 \mathrm{~mm}$ in diameter (Fig. 2). The histopathological and immunohistochemical findings are summarized in Figs. 3 and 4, respectively. As shown in Fig. 3A and B, the focus of the invasive carcinoma was located at the periphery of the IPMN. The pathological diagnosis of the invasive carcinoma was anaplastic giant-cell carcinoma. The IPMN cells exhibited mucin 2 immunoreactivity (anti-MUC2 monoclonal mouse antibody; dilution, 1:20; cat. no. 555926; BD Biosciences) (Fig. 4A). The mono- and multinuclear cells of the ACP exhibited similar immunoprofiles (monoclonal mouse antibody against cytokeratin 7; dilution, 1:100; cat. no. M7014; Dako) (Fig. 4B). The mindbomb E3 ubiquitin protein ligase 1 (MIB-1; Ki-67) mean labeling index was $\sim 5 \%$ around the IPMN (Fig. 4C), and $25 \%$ in sites distant from the IPMN (MIB-1 monoclonal mouse antibody; dilution, 1:150; cat. no. M7240; Dako). However, a part of the IPMN with 

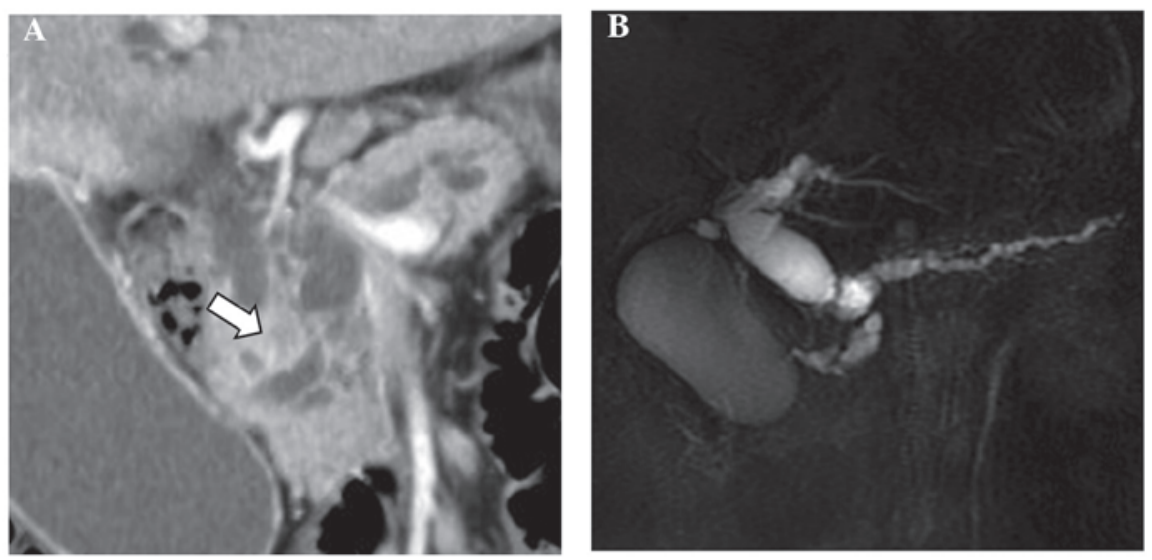

Figure 1. (A) Coronal section of a computed tomography scan revealing an irregular mass (arrow) in the pancreatic head exhibiting lower enhancement relative to the non-tumor pancreatic parenchyma in the arterial-dominant phase. Cysts located in the inferior part of the irregular mass were observed. (B) Magnetic resonance cholangiopancreatography showing stenosis of the main pancreatic and common bile ducts, caused by the mass-neighboring cysts.

high-grade dysplasia exhibited continuous transition to invasive carcinoma, lacked polarity, and displayed stratified and pleomorphic nuclei (Fig. 3C); these lesions had an MIB-1 index of $\sim 80 \%$. The final pathological diagnosis was IPMN (intestinal type, involving the main and branch duct system), with an associated invasive carcinoma of the anaplastic giant-cell type. The tumor was classified as stage III (5). The patient was prescribed S-1 for 6 months following surgery, and her postoperative course was uneventful. The patient remains disease-free at 18 months postoperatively.

\section{Discussion}

ACPs are rare and highly aggressive tumors (1), with common symptoms including weight loss, fatigue, loss of appetite, abdominal pain, nausea and vomiting. Three histological variants of anaplastic carcinoma, namely the spindle-, pleomorphic- and giant-cell types, have been described (5). These carcinomas are classified as undifferentiated (anaplastic), whereas anaplastic carcinoma with osteoclast-like giant cells (OGCs) is classified as a subtype of invasive ductal carcinoma (6). Preoperative diagnosis of ACP is difficult; on imaging studies, ACPs are usually detected as large, moderately hypervascular, exophytic tumors, with large areas of necrosis (1). Anaplastic foci may be identified in ductal adenocarcinomas and in ectopic pancreatic tissue, although they are not the dominant pattern of growth (7). It was previously demonstrated that OGCs are positive for the histiocytic marker CD68, without reactivity for epithelial markers (8). In the present case, the tumor giant cells were positive for cytokeratin 7 and negative for CD68, suggesting an epithelial origin.

ACP coexisting with IPMN is extremely rare, with only 6 previously reported cases (4). However, those cases, in which there were no foci of IPMN dedifferentiation to ACP, were considered to be ACP coexisting with IPMN rather than ACP originating in IPMN. PDAC may develop in a pancreatic duct independently from an $\operatorname{IPMN}(9,10)$. When PDAC originates in the vicinity of an IPMN, the distinction between PDAC derived from the IPMN and PDAC concomitant with the IPMN may occasionally be difficult. Definitions of these

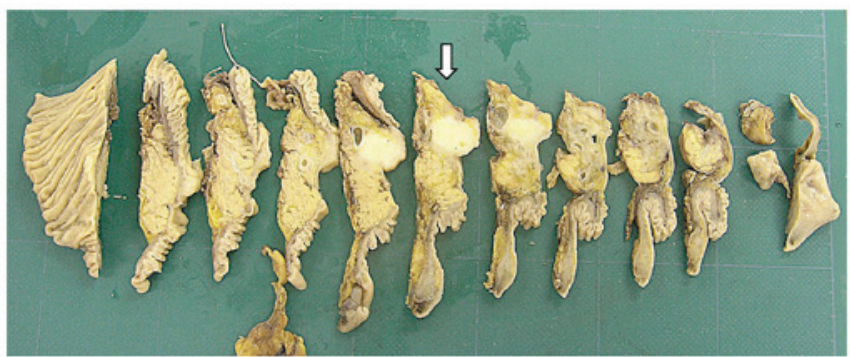

Figure 2. Serial sections of the pancreatic tumors. The IPMN-involved ducts appear dilated and the cut surface of the invasive tumor (arrow) is solid and whitish yellow. IPMN, intraductal papillary mucinous neoplasm.

conditions were recommender by the Japan Pancreas Society, mainly with regard to the topological association and histological transition between IPMN and PDAC $(5,10)$. Due to the evidence of 'budding and intruding' of the IPMN into the ACP, our case is considered to be an ACP originating in an IPMN. In our case, the foci where the IPMN dedifferentiated to ACP were located at the periphery of the IPMN. The primary focus of malignant invasive tumors is usually located in the center of the developed mass; however, in the present case, several other foci were present, which were also suspected as lesions where IPMN dedifferentiated to ACP. Therefore, the dedifferentiation was possibly multicentric. At the site distant from the IPMN, the anaplastic cancer cells became larger and exhibited a higher MIB-1 index, suggesting further dedifferentiation with increased proliferation and infiltration of the tumor cells. In addition, the frequent giant cells positive for cytokeratin 7 support the hypothesis of an epithelial origin and dedifferentiation of the IPMN.

In conjunction with the finding that a subset of IPMN-associated invasive carcinomas tend to have a better prognosis compared with adenocarcinomas arising in the setting of pancreatic intraepithelial neoplasia (PanIN), an emerging consensus suggests that there are two major pathways leading to the development of invasive carcinoma in the pancreas: One that is more aggressive (via PanIN and pancreatobiliary-type IPMN precursors) and a second that is more indolent (via intestinal-type IPMN precursors) (6). It is 
A

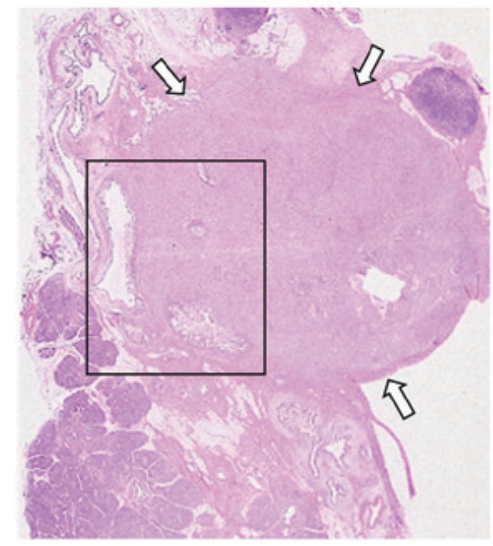

C

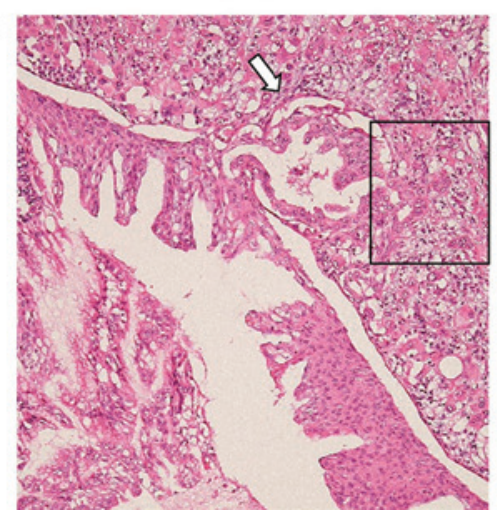

B

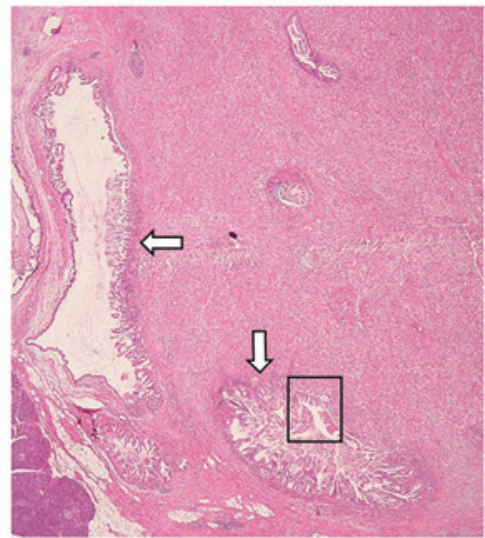

D

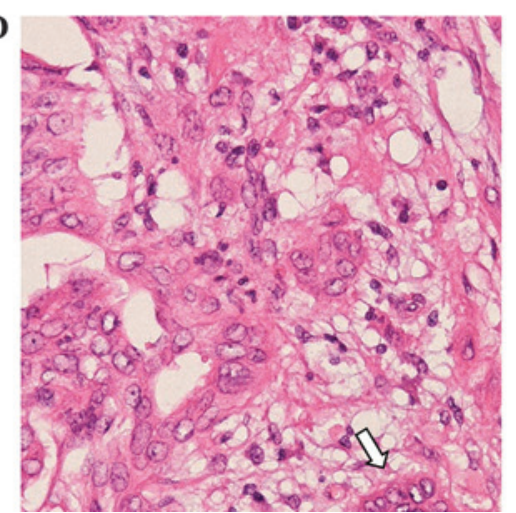

Figure 3. Hematoxylin and eosin-stained sections of the tumor. (A) The arrows indicate the invasive carcinoma. Irregularly dilated abnormal ducts involved by the intraductal papillary mucinous neoplasm (IPMN) may be seen in and beneath the invasive cancer component; magnification, $\mathrm{x} 1$. (B) Area of the open square shown in (A); magnification, x10. The arrows indicate the main pancreatic duct involved by the IPMN. (C) Area of the open square shown in (B); magnification, $x 40$. Photomicrograph of the intestinal-type IPMN with high-grade dysplasia. Part of the IPMN was budding and intruding into the surrounding anaplastic lesion (arrow). (D) Area of the open square shown in (C); magnification, x100. The IPMN cells exhibited continuous transition to invasive anaplastic carcinoma. The arrow indicates bizarre-appearing giant cells.
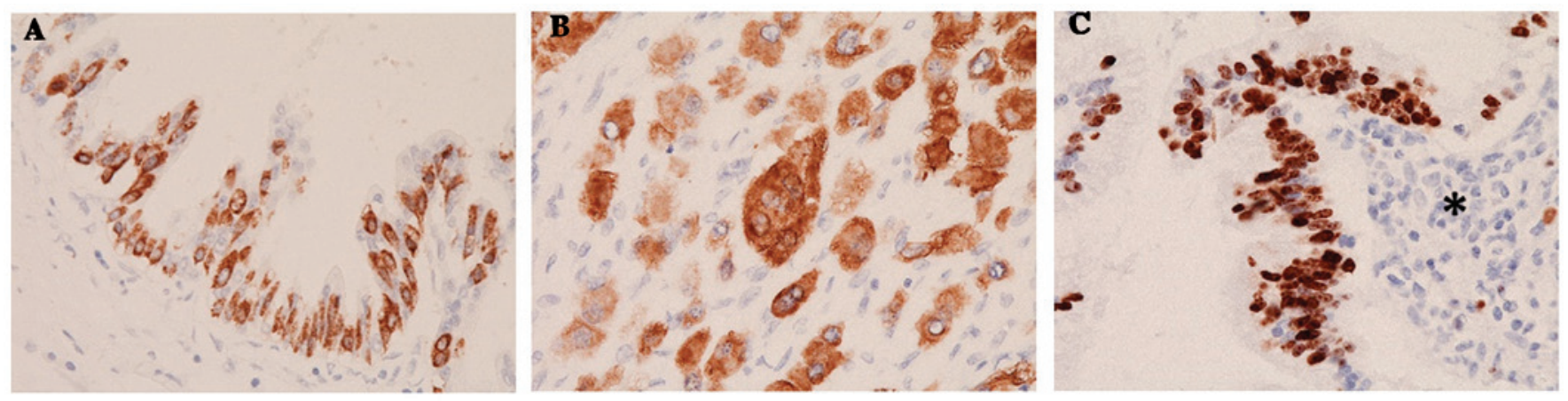

Figure 4. Immunohistochemical analysis of the pancreatic tumor. (A) The intraductal papillary mucinous neoplasm (IPMN) cells exhibited mucin 2 immunoreactivity. (B) The mononuclear and multinuclear cells of the anaplastic carcinoma (ACP) exhibited immunoreactivity for cytokeratin 7. (C) A higher mindbomb E3 ubiquitin protein ligase 1 labeling ratio was observed in the IPMN with high-grade dysplasia compared with the ACP (asterisk).

possible that these major pathways to invasive carcinoma are applicable to ACP as well. Thus, the prognosis of ACP derived from IPMN is not necessarily poor.

In conclusion, ACP is associated with a poorer survival compared with invasive PDAC. Irrespective of the treatment, the prognosis for this type of tumor remains grave, due to its aggressive nature and rapid recurrence. IPMNs in the clinical field of invasive pancreatic carcinoma should not be overlooked and, in cases of ACP derived from IPMN, immunohistochemistry should be applied to determine whether they are of the intestinal type histologically. The case presented herein may enable a better understanding of the pathogenesis of ACP.

\section{Acknowledgements}

We would like to thank Japan Clinical Laboratories, Inc. for fostering constructive discussions and scientific understanding with different medical backgrounds. All the authors have read and approved this manuscript. 


\section{References}

1. Paal E, Thompson DL, Frommelt RA, Przygodzki RM and Heffes CS: A clinicopathologic and immunohistochemical study of 35 anaplastic carcinomas of the pancreas with review of the literature. Ann Diagn Pathol 5: 129-140, 2001.

2. Schnelldorfer T, Sarr MG, Nagorney DM, Zhang L, Smyrk TC, Qin R, Chari ST and Farnel MB: Experience with 208 resections for intraductal papillary mucinous neoplasm of the pancreas. Arch Surg 143: 639-646, 2008.

3. Tanaka M, Fernández-del Castillo C,Adsay V, Chari S, Falconi M, Jang JY, Kimura W, Levy P, Pitman MB, Schmidt CM, et al: International consensus guidelines 2012 for the management of IPMN and MCN of the pancreas. Pancreatology 12: 183-197, 2012.

4. Shon TA, Yeo CJ, Cameron JL, Hruban RH, Fukushima N, Campbell KA and Lillemoe KD: Intraductal papillary mucinous neoplasms of the pancreas: An updated experience. Ann Surg 239: 788-797; discussion 797-799, 2004.

5. Japan Pancreas Society: General Rules for the Study of Pancreatic Cancer. 6th edition. Kanehara Shuppan, Tokyo, pp1-13, 2013
6. Bosman FT, Carneiro F, Hruban RH and Theise ND (eds): WHO classification of tumors of the digestive system. In: WHO Classification of Tumours. Vol. 3. 4th edition. IARC Press, Lyon, 2010.

7. Roshe J, Del Buono E, Domenico D and Colturi TJ: Anaplastic carcinoma arising in ectopic pancreas located in the distal esophagus. J Clin Gastroenterol 22: 242-244, 1996.

8. Molberg KH, Heffes C, Delgado R and Albores-Saavedra J: Undifferentiated carcinoma with osteoclast-like giant cells of the pancreas and periampullary region. Cancer 82: 1279-1287, 1998

9. Tanaka M: Controversies in the management of pancreatic IPMN. Nat Rev Gastroenterol Hepatol 8: 56-60, 2011.

10. Yamaguchi K, Ohuchida J, Ohtsuka T, Nakano K and Tanaka M: Intraductal papillary-mucinous tumor of the pancreas concomitant with ductal carcinoma of the pancreas. Pancreatology 2: 484-490, 2002. 